\title{
Protective effect of probiotics in the treatment of infantile eczema
}

\author{
RONG-JUN LIN ${ }^{1}$, LI-HUA QIU ${ }^{2}$, REN-ZHENG GUAN ${ }^{1}$, SU-JUAN HU ${ }^{2}$, \\ YING-YING LIU ${ }^{2}$ and GUANG-JUN WANG ${ }^{3}$ \\ ${ }^{1}$ Department of Pediatrics, The Affiliated Hospital of Qingdao University, Qingdao, Shandong 266071; \\ ${ }^{2}$ Department of Pediatrics, The Maternal-Child Healthcare Center of Qingdao, Qingdao, Shandong 266035; \\ ${ }^{3}$ Department of Pediatrics, Shandong Traffic Hospital, Jinan, Shandong 250031, P.R. China
}

Received May 14, 2014; Accepted December 18, 2014

DOI: $10.3892 /$ etm.2015.2299

\begin{abstract}
The aim of the present study was to provide evidence for the application of probiotics in the prevention and treatment of infantile eczema by exploring changes in the intestinal Bifidobacteria levels and the Scoring Atopic Dermatitis (SCORAD) index prior and subsequent to treatment with probiotics in infants with eczema. A total of 40 infants with eczema were randomly divided into treatment and control groups. Prior and subsequent to the treatment, the SCORAD index was evaluated and the content of Bifidobacterium bifidum in the stool of each infant in the two groups was quantified using 16S rRNA/DNA quantitative polymerase chain reaction analysis. After four weeks of treatment with B. bifidum triple viable capsules, the levels of $B$. bifidum increased sharply $(\mathrm{P}<0.05)$ and the SCORAD index was notably reduced $(\mathrm{P}<0.05)$ as compared with the values prior to treatment. By contrast, neither the content of B. bifidum nor the SCORAD index changed significantly in the control group after four weeks $(\mathrm{P}>0.05)$. Following treatment, the levels of $B$. bifidum in the stools of the treatment group were significantly higher than those in the stools of the control group $(\mathrm{P}<0.05)$, and the SCORAD index was significantly lower than that of the control group $(\mathrm{P}<0.05)$. In conclusion, probiotic supplementation has a positive effect on the prevention and treatment of infantile eczema.
\end{abstract}

\section{Introduction}

Eczema is an allergic skin disease that occurs in the superficial dermis and epidermis. The condition can be caused by a number of factors, including genetic defects and foreign substances (1). Eczema is most common in infants and young children and all ethnicities can be affected (2). The

Correspondence to: Dr Rong-Jun Lin, Department of Pediatrics, The Affiliated Hospital of Qingdao University, 59 Haier Road, Qingdao, Shandong 266071, P.R. China

E-mail: rongjunlin@yeah.net

Key words: Bacillus bifidus, probiotics, infants, eczema disease may seriously impact the growth and development of infants. Certain infants with severe eczema are vulnerable to secondary bacterial or viral infections, causing serious complications (3).

Since $35-50 \%$ of infantile eczema cases are caused by food allergy, avoiding food allergens and using emollients, anti-allergy therapies and local or systemic hormones are currently regarded as the main treatments of infantile eczema (4). In recent years, studies have found that probiotics can modulate the body's immune status and improve the gut barrier function that contributes to the prevention and treatment of infantile eczema, particularly for those cases caused by food allergies (5-7). Supplementation with probiotics may thus be beneficial for the treatment of infantile eczema (8). A randomized, double-blind, placebo-controlled study (9) suggested that supplementation of certain specific probiotics could alleviate the symptoms of eczema, such as itching, insomnia, erythema, exudation and skin xerochase, and reduce the loss of skin moisture. Probiotics may therefore maintain the complete barrier function of the skin and reduce bacterial infection opportunities; however, not all probiotics are effective for the treatment of eczema, and successful treatment is dependent on the species of probiotics, the colonization site in the gastrointestinal tract and the roles of the different species (10). The dose of additional probiotics may also be an influencing factor. Despite this, the effect of probiotics in the clinical prevention and treatment of eczema lacks medical evidence. In order to investigate the clinical efficacy of probiotics in the treatment of infantile eczema, Bifidobacterium bifidum was selected as a representative probiotic species in the present study, and the levels of B. bifidum in the intestinal tract and the Scoring Atopic Dermatitis (SCORAD) index were evaluated prior and subsequent to a four-week regimen with $B$. bifidum triple viable capsules for the treatment of infantile eczema.

\section{Materials and methods}

Subjects. Forty infants with newly diagnosed eczema, who were patients in the Department of Pediatrics in the Affiliated Hospital of Qingdao University Medical College (Qingdao, China) between December 2010 and March 2011, were enrolled in this study. All the subjects met the diagnostic criteria for eczema (11). Written/verbal consent was obtained 
from the patients' guardians. The present study was approved by the Medical Ethics Committee of the Affiliated Hospital of Qingdao University (Qingdao, China). The 40 patients included 21 males and 19 females, with 26 patients aged less than one year and 14 patients aged between one and three years. The 40 infants were randomly divided into treatment and control groups. The treatment group $(n=20)$, which included nine males and 11 females with a mean age of $11.45 \pm 7.87$ months, was orally administered $B$. bifidum triple viable capsules (Shanghai Sine Pharmaceutical, Corp, Ltd., Shanghai, China) for four weeks with a dosage of one capsule three times per day. The control group $(n=20)$, which included 12 males and eight females with a mean age of $12.26 \pm 8.31$ months, did not receive any special treatment and were not adminstered a placebo drug. The age, gender, disease duration and degree of eczema flare of the two groups showed no significant differences. The 40 infants were treated with anti-allergy therapy and dietary guidance. None of the enrolled children had been treated with any antibiotics, probiotics or other drugs and food at least two weeks prior to the start of this study. Children suffering from pneumonia, capillary bronchitis and other diseases or who had been treated with antibiotics or hormones during the experimental process were excluded from this study. Prior to treatment (baseline) and after four weeks of treatment, stool samples were collected from the patients in the two groups with sterile plastic tubes and stored at $-80^{\circ} \mathrm{C}$ in the refrigerator.

Preparation of intestinal bacterial DNA. All specimens stored at $-80^{\circ} \mathrm{C}$ were thawed at room temperature. The bacterial DNA was extracted from each 0.2-g stool sample with a fecal genomic DNA extraction kit (Tiangen Biotech (Beijing) Co., Ltd., Beijing, China) according to the manufacturer's instructions.

Polymerase chain reaction (PCR) primer design. The 16S rRNA sequence of $B$. bifidum was obtained from GenBank (http://www.ncbi.nlm.nih.gov/genbank/). The following gene primers were used: Forward, 5'-GATTCTGGCTCA GGATGAACGC-3'; reverse, 5'-CTGATAGGACGCGAC CCCAT-3'. The product size was $230 \mathrm{bp}$. Primers were designed with Primer 5.0 software (Premier Biosoft International, Palo Alto, CA, USA) according to the primer design principles and synthesized by Shanghai Sangon Biotech. Co., Ltd. (Shanghai, China). To verify the specificity of the primers, the primer sequences were also aligned with the gene in the Basic Local Alignment Search Tool gene library (http://blast.ncbi.nlm.nih. gov/Blast.cgi).

$P C R$ reaction. A 2- $\mu 1$ DNA sample was used as the $\mathrm{PCR}$ template with a PCR kit from Tiangen Biotech (Beijing) Co., Ltd. The total PCR mixture $(25 \mu \mathrm{l})$, containing $12.5 \mu \mathrm{l} 2 \mathrm{X}$ Taq PCR Master Mix solution, $0.5 \mu \mathrm{l}$ forward primer (25 $\mu \mathrm{mol} / \mathrm{l})$, $0.5 \mu 1$ reverse primer $(25 \mu \mathrm{mol} / 1), 2 \mu \mathrm{l}$ DNA template and sterile water, was run on a GeneAmp ${ }^{\circledR}$ PCR system 9600 (Invitrogen Life Technologies, Carlsbad, CA, USA) with a program of $95^{\circ} \mathrm{C}$ for $5 \mathrm{~min}, 35$ cycles of $95^{\circ} \mathrm{C}$ for $15 \mathrm{sec}, 60^{\circ} \mathrm{C}$ for $60 \mathrm{sec}$ and $72^{\circ} \mathrm{C}$ for $45 \mathrm{sec}$, with a final extension step of $72^{\circ} \mathrm{C}$ for $10 \mathrm{~min}$. The PCR products were then analyzed by agarose gel electrophoresis.
Quantitative PCR (qPCR). A 20- $\mu$ l qPCR system (Applied Biosystems $^{\circledR}$, Invitrogen Life Technologies, Foster City, CA, USA) containing $10 \mu \mathrm{l} 2 \mathrm{X} \mathrm{SYBR}{ }^{\circledR}$ Green qPCR Master Mix, $1 \mu \mathrm{l}$ forward primer $(10 \mu \mathrm{mol} / 1), 1 \mu \mathrm{l}$ reverse primer (10 $\mu \mathrm{mol} / \mathrm{l}), 1 \mu \mathrm{l}$ DNA template and $7 \mu \mathrm{l}$ sterile water was used for the quantitative analysis. The qPCR was run on an ABI StepOnePlus ${ }^{\mathrm{TM}}$ Real-Time PCR analyzer (Applied Biosystems, Foster City, CA, USA) with the following program: $95^{\circ} \mathrm{C}$ for $5 \mathrm{~min}, 35$ cycles of $95^{\circ} \mathrm{C}$ for $15 \mathrm{sec}, 60^{\circ} \mathrm{C}$ for $60 \mathrm{sec}, 72^{\circ} \mathrm{C}$ for $45 \mathrm{sec}$ and $87^{\circ} \mathrm{C}$ for $5 \mathrm{sec}$ (for accumulating fluorescence), and a final extension step of $72^{\circ} \mathrm{C}$ for $10 \mathrm{~min}$. The initial $\mathrm{Ct}$ value was then obtained from the fluorescence curve.

Standard curve and quantitative analysis. B. bifidum freeze-dried powder strains (offered by the China General Microbiological Culture Collection Center, Beijing, China) were inoculated in MRS liquid medium (Yangsheng chem, Shanghai, China) and incubated in anaerobic conditions of $90 \% \mathrm{~N}_{2}$ and $10 \% \mathrm{CO}_{2}$ for $24 \mathrm{~h}$ at $37^{\circ} \mathrm{C}$. The concentration of the DNA extracted from the culture was measured by a spectrophotometer. The DNA was then serially diluted in a 10 -fold manner to concentrations of $1 \times 10^{7}$ to $1 \times 10^{3} \mathrm{ng} / \mu \mathrm{l}$ and used as templates for the qPCR to make a standard curve. Based on this standard curve, the concentrations of the intestinal bacterial DNA could be obtained.

SCORAD index. The 40 infants with eczema enrolled in this study underwent a SCORAD evaluation at the first outpatient appointment and again four weeks later, during the follow-up outpatient appointment. The SCORAD evaluation was carried out on an outpatient basis to assess factors including eczema area, severity of eczema and the patients' assessment of their condition, and calculated by the following formula: $\mathrm{SCORAD}=\mathrm{A} / 5+7 \mathrm{~B} / 2+\mathrm{C}$, in which $\mathrm{A}$ was the lesion area score, B was the lesion severity score evaluated by physicians (out of a possible six levels) and $\mathrm{C}$ was symptom severity score (itching and insomnia) evaluated by the parents.

Statistical analysis. Statistical analysis was performed with SPSS version 17.0 software (SPSS, Inc., Chicago, IL, USA). The quantitative data of $B$. bifidum DNA are as presented as the mean \pm standard deviation following logarithmic transformation. Comparisons between the groups were performed with a Student's t-test, and $\mathrm{P}<0.05$ was considered to indicate a statistically significant difference.

\section{Results}

B. bifidum supplementation significantly enhances the levels of B. bifidum in the intestine. The levels of B. bifidum in the infants' stool samples were detected by qPCR using an absolute quantification method. Prior to the treatment, no significant difference was found in the bacterial DNA level of the stool samples between the treatment and control groups $(\mathrm{P}>0.05)$; however, after four weeks of treatment with $B$. bifidum triple viable capsules for the infants in the treatment group, the levels of B. bifidum in the infants' stool samples were observed to be markedly increased $(\mathrm{P}<0.05)$ and were significantly higher than those of the control group $(\mathrm{P}<0.05)$ (Table I). 
Table I. Bifidobacterium bifidum content in the stool samples from the two groups of children with eczema.

\begin{tabular}{lccc}
\hline Group & Pretreatment (copies/g stool) & Post-treatment (copies/g stool) & t-value \\
\hline Treatment & $6.50 \pm 0.34$ & $9.19 \pm 0.28$ & 27.13 \\
Control & $6.49 \pm 0.31$ & $6.52 \pm 0.28$ & 0.34 \\
t-value & 0.15 & 30.18 & - \\
P-value & 0.88 & $<0.001$ & - \\
\hline
\end{tabular}

Data are presented as the mean \pm standard deviation.

Table II. SCORAD index of the two groups of children with eczema.

\begin{tabular}{|c|c|c|c|c|}
\hline Group & Pretreatment & Post-treatment & t-value & P-value \\
\hline Treatment & $24.90 \pm 13.36$ & $15.95 \pm 7.58$ & 2.61 & 0.01 \\
\hline Control & $25.20 \pm 11.76$ & $22.90 \pm 12.51$ & 0.60 & 0.55 \\
\hline t-value & 0.08 & 2.13 & - & - \\
\hline P-value & 0.94 & 0.04 & - & - \\
\hline
\end{tabular}

Data are presented as the mean \pm standard deviation. SCORAD, Scoring Atopic Dermatitis.

B. bifidum supplementation significantly reduces the SCORAD index of infants with eczema. Prior to the treatment with $B$. bifidum, no significant difference was found in the SCORAD index between the two groups. After four weeks of treatment with $B$. bifidum, however, the SCORAD index was reduced markedly $(\mathrm{P}<0.05)$ and was significantly lower than that of the control group $(\mathrm{P}<0.05)$ (Table II).

\section{Discussion}

In the United States, 10-20\% of the infant population suffers with eczema. It is well known that recurrent skin lesions, itching and secondary infection are seriously harmful to the health of infants and young children. Eczema may be the first symptom of allergic diseases in children, and the condition can develop into a number of such allergic diseases, including allergic asthma, rhinitis and chronic urticaria; these diseases can impose a significant economic and psychological burden on the children and their families (12). In addition to genetic factors and food allergies, eczema may be associated with immature intestinal immune function and the colonization of intestinal flora (13-15).

Normal intestinal flora plays an important role in maintaining human health and can promote the development of intestinal lymph nodes and cause the increased production of secretory immunoglobulin, thereby increasing the resistance of the body to disease $(16,17)$. B. bifidum and lactic acid bacteria are considered to be two important human probiotics $(6,18)$. These bacteria colonize in the intestinal tract and resist exogenous pathogens, so as to enhance the immune status of the body. B. bifidum and other probiotics also have a number of other roles, including improving the barrier function of the intestinal immune system, regulating the immune response and reducing the production of inflammatory cytokines and the inflammatory response (7).
In the present study, it was found that treatment with $B$. bifidum could markedly enhance the levels of B. bifidum in the stools and alleviate the eczema in the infants. It has previously been reported that the number of B. bifidum is typically lower in the stools of infants with eczema (19). With regard to the prevention and treatment of the condition, Moro et al (20) found that the administration of probiotics could prevent the occurrence of eczema, while Toh et al (21) reported that probiotics had a preventive and therapeutic role on eczema and other allergic diseases. These studies suggest that the intestinal flora of children with eczema is disordered and that probiotics have a certain effect on the prevention and treatment of eczema.

With improvements in medical standards and understanding, considerable attention has been focused on the role of probiotics in the prevention and treatment of infantile eczema. Probiotics have been demonstrated to possess an immunomodulatory function and are able to improve the intestinal barrier function and reduce the inflammatory response (22). In addition, it was found in a previous study that no adverse reactions were reported when probiotics were used in a normal population of infants and young children (23). Despite this, there remains a lack of clinical evidence regarding the safety of the long-term use of probiotics and the standard prescription for treatment (24). Furthermore, a consensus generated by the Food and Agriculture Organization of the United Nations and the World Health Organization in 2006 emphasized that any probiotic strains used for humans must meet strict standards (25).

In conclusion, the findings of the present study have indicated that the short-term administration of probiotics can be regarded as an effective treatment for infantile eczema; however, further clinical investigation into the long-term use and side effects of probiotics is required prior to the application of long-term probiotic supplementation as a clinical strategy. 


\section{References}

1. Bieber T: Atopic Dermatitis. Ann Dermatol 22: 125-137, 2010.

2. Baron SE, Cohen SN, Archer CB; British Association of Dermatologists and Royal College of General Practitioners: Guidance on the diagnosis and clinical management of atopic eczema. Clin Exp Dermatol 37 (Suppl 1): 7-12, 2012.

3. Ozdemir O: Various effects of different probiotic strains in allergic disorders: an update from laboratory and clinical data. Clin Exp Immunol 160: 295-304, 2010.

4. Urisu A, Ebisawa M, Mukoyama T, et al: Japanese guideline for food allergy. Allergol Int 60: 221-236, 2011.

5. Jensen MP, Meldrum S, Taylor AL, et al: Early probiotic supplementation for allergy prevention: long-term outcomes. J Allergy Clin Immunol 130: 1209-1211, 2012.

6. Ouwehand AC: Antiallergic effects of probiotics. J Nutr 137 (3 Suppl 2): 794S-797S, 2007.

7. Ozdemir O: Any benefits of probiotics in allergic disorders? Allergy Asthma Proc 31: 103-111, 2010.

8. Fiocchi A, Burks W, Bahna SL, et al; WAO Special Committee on Food Allergy and Nutrition: Clinical Use of Probiotics in Pediatric Allergy (CUPPA): a world allergy organization position paper. World Allergy Organ J 5: 148-167, 2012.

9. Guéniche A, Hennino A, Goujon C, et al: Improvement of atopic dermatitis skin symptoms by Vitreoscilla filiformis bacterial extract. Eur J Dermatol 16: 380-384, 2006.

10. Abrahamsson TR, Jakobsson HE, Andersson AF, et al: Low diversity of the gut microbiota in infants with atopic eczema. J Allergy Clin Immunol 129: 434-440, 2012.

11. Fritsch PO and Reider N: Other eczematous eruptions. In: Dermatology. Bolognila JL, Jorizzo JL and Rapini RP (eds). 2nd edition. Elsevier, Philadelphia, PA, pp201-202, 2008.

12. Garrett JP, Apter AJ, Hoffstad O, et al: Asthma and frequency of wheeze: risk factors for the persistence of atopic dermatitis in children. Ann Allergy Asthma Immunol 110: 146-149, 2013.

13. Tang ML: Probiotics and prebiotics: immunological and clinical effects in allergic disease. Nestle Nutr Workshop Ser Pediatr Program 64: 219-238, 2009.

14. Kuitunen M, Kukkonen K, Juntunen-Backman K, et al: Probiotics prevent IgE-associated allergy until age 5 years in cesarean-delivered children but not in the total cohort. J Allergy Clin Immunol 123: 335-341, 2009.
15. Yan F and Polk DB: Probiotics and immune health. Curr Opin Gastroenterol 27: 496-501, 2011.

16. Fontana L, Bermudez-Brito M, Plaza-Diaz J, et al: Sources, isolation, characterisation and evaluation of probiotics. $\mathrm{Br}$ J Nutr 109 Suppl 2: S35-S50, 2013.

17. Rastall RA: Bacteria in the gut: friends and foes and how to alter the balance. J Nutr 134 (8 Suppl): 2022S-2026S, 2004.

18. Hwang JS, Im CR and Im SH: Immune disorders and its correlation with gut microbiome. Immune Netw 12: 129-138, 2012.

19. Young SL, Simon MA, Baird MA, et al: Bifidobacterial species differentially affect expression of cell surface markers and cytokines of dendritic cells harvested from cord blood. Clin Diagn Lab Immunol 11: 686-890, 2004.

20. Moro G, Arslanoglu S, Stahb B, et al: A mixture of prebiotic oligosaccharides reduces the incidence of atopic dematitis during the first six months of age. Arch Dis Child 91: 814-819, 2006.

21. Toh ZQ, Anzela A, Tang ML and Licciardi PV: Probiotic therapy as a novel approach for allergic disease. Front Pharmacol 3: 171, 2012.

22. Gore C, Custovic A, Tannock GW, et al: Treatment and secondary prevention effects of the probiotics Lactobacillus paracasei or Bifidobacterium lactis on early infant eczema: randomized controlled trial with follow-up until age 3 years. Clin Exp Allergy 42: 112-122, 2012.

23. Agarwal R, Sharma N, Chaudhry R, et al: Effects of oral Lactobacillus GG on enteric microflora in low-birth-weight neonates. J Pediatr Gastroenterol Nutr 36: 397-402, 2003.

24. Pan SJ, Kuo CH, Lam KP, et al: Probiotics and allergy in children - an update review. Pediatr Allergy Immunol 21: e659-e666, 2010.

25. Food and Agriculture Organization of the United Nations; World Health Organization; Joint FAO/WHO Expert Consultation on Evaluation of Health and Nutritional Properties of Probiotics in Food including Powder Milk with Live Lactic Acid Bacteria; Joint FAO/WHO Working Group on Drafting Guidelines for the Evaluation of Probiotics in Food: Probiotics in Food: Health and Nutritional Properties and Guidelines for Evaluation. FAO/WHO, Rome, 2006. 